\title{
5 Entwicklung einer Kostensensiblen Leitlinie zu Medikamente-freisetzenden Stents bei Patienten mit einer koronaren Herzkrankheit
}

\author{
Petra Schnell-Inderst, Daniela Gartner-Freyer, Janine Biermann, \\ Kirstin Börchers, Anja Neumann und Jürgen Wasem
}

\subsection{Hintergrund}

Begrenzte Ressourcen, ein steigender Bedarf an Gesundheitsleistungen aufgrund der Alterung der Bevölkerung und ein stetiger Strom häufig teurer medizinischer Innovationen bilden den Hintergrund für Diskussionen um Priorisierung und Rationierung von Gesundheitsleistungen in der GKV. Im Unterschied zu Anreizen für implizite Formen der Leistungsbegrenzung wie Fallpauschalen und Arzneimittelbudgets könnte ein Übergang zu expliziteren Formen der Leistungsbegrenzung wie Kostensensiblen Leitlinien (KSLL) für Arzt und Patient zu mehr Transparenz über Kriterien von Leistungseinschränkungen führen und das Arzt-Patient-Verhältnis entlasten. Rein klinisch orientierte Leitlinien unterstützen Arzt und Patient bei der Abwägung von Zusatznutzen und Schaden durch neue Technologien, differenzieren hierbei zwischen verschiedenen Patientengruppen, soweit dies für Prävention, Diagnostik und Therapie relevant ist, und berücksichtigen bei den Behandlungsempfehlungen den nationalen Versorgungskontext. Durch KSLL könnte nun als weiteres Element in diese Abwägung die gesellschaftliche Zahlungsbereitschaft bzw. die Zahlungsbereitschaft des Leistungsträgers einbezogen werden (ausführlicher im Kapitel 3: Erstellung Kostensensibler Leitlinien: methodologische Überlegungen aus gesundheitsökonomischer Sicht). Im Folgenden wird am Fallbeispiel von Medikamente-freisetzenden Koronarstents (Drug Eluting Stents, DES) untersucht, ob es möglich und praktikabel ist, Kostensensible Leitlinien zu 
5 Entwicklung einer Kostensensiblen Leitlinie zu Medikamente-freisetzenden Stents bei Patienten mit einer koronaren Herzkrankheit

erstellen. Mögliche Schwierigkeiten bei diesem Prozess sollen identifiziert werden. Eine kurze Einführung zum Krankheitsbild und eine Beschreibung der Anwendung und des Wirkprinzips von DES und konventionellen Metallstents (Bare Metal Stents, BMS) bei der Koronarintervention findet sich in der Einführung zu Kapitel 6.

\subsection{Methodik}

Im ersten Schritt wurde in einer systematischen Recherche eine auf den deutschen Versorgungskontext übertragbare evidenzbasierte Leitlinie mit ausreichender methodischer Qualität zum Einsatz von DES bei perkutanen Koronarinterventionen (PCI) im Vergleich zu BMS ausgewählt. Die Evidenz zur klinischen Effektivität, die Abwägung von zusätzlichem Nutzen und Schaden für verschiedene Patientengruppen, die daraus abgeleiteten Empfehlungen und die Berücksichtigung von Kosteneffektivitätsaspekten wurden dargestellt. Im zweiten Schritt wurde ein systematischer Literaturreview zur Kosteneffektivität von DES für Subgruppen erstellt. Die Daten zu dem Studiendesign, der methodischen Qualität und den Ergebnissen der Studien wurden extrahiert und zur Analyse methodischer Aspekte bei der Erstellung von KSLL verwendet. In einem dritten Schritt wurde als Beispiel für eine existierende KSLL die „Guidance“ zu DES des National Institutes for Health and Clinical Excellence (NICE) 2008 dargestellt, die nach Redaktionsschluss der Leitlinienrecherche 2007 erschienen war.

\subsubsection{Systematische literaturrecherche zu klinischen Leitlinien zu DES}

In medizinischen Datenbanken und auf Internetseiten von Leitlinienorganisationen sowie medizinischen Fachgesellschaften (s. Tab. 20) wurde nach nationalen und internationalen Leitlinien mit den folgenden Einschlusskriterien gesucht: Es handelt sich um eine Leitlinie zum Einsatz von DES bei PCI, die Leitlinie ist für Deutschland oder ein europäisches oder außereuropäisches Land bestimmt, für das eine Übertragbarkeit auf Deutschland angenommen werden kann. Zwei Bearbeiter selektierten unabhängig voneinander die Leitlinien anhand von Titeln und Abstracts. Die ausgewählten Leitlinien wurden einer Qualitätsbewertung anhand des Bewertungsinstruments DELBI unterzogen. Zusätzliche Auswahlkriterien waren die Übertragbarkeit auf den Kontext des deutschen Gesundheitssystems und die Aktualität der Leitlinie. Die Auswahl erfolgte wiederum unabhängig durch zwei Bearbeiter. Die Empfehlungen und die Evidenz zur klinischen Effektivität wurden extrahiert. 
Tab. 20 Quellen für die Leitlinienrecherche

\begin{tabular}{ll}
\hline $\begin{array}{l}\text { Leitlinienorganisationen und } \\
\text { medizinische Fachgesellschaften }\end{array}$ & $\begin{array}{l}\text { Agency for Healthcare Research and Quality (AHRQ): } \\
\text { National Guideline Clearinghouse (NGC) }\end{array}$ \\
\hline Scottish Intercollegiate Guidelines Network (SIGN) \\
\hline Health Evidence Bulletins - Wales \\
\hline Guidelines International Network (GIN) \\
\hline Guidelines Advisory Committee (GAC) \\
\hline National Institute for Health and Clinical Excellence (NICE) \\
\hline New Zealand Guidelines Group (NZGG) \\
\hline American Heart Association (AHA) \\
\hline European Society for Cardiology \\
\hline Deutsche Gesellschaft für Kardiologie (DGK) \\
\hline $\begin{array}{l}\text { Arbeitsgemeinschaft der Wissenschaftlichen Medizinischen } \\
\text { Fachgesellschaften (AWMF) }\end{array}$ \\
\hline Bundesärztekammer (BÄK) \\
\hline $\begin{array}{l}\text { Medizinisches Wissensnetzwerk evidence.de der Universität } \\
\text { Witten/Herdecke }\end{array}$ \\
\hline Medline \\
\hline bibliographische Datenbank
\end{tabular}

\subsubsection{Literaturreview zur Kosteneffektivität von DES in klinisch relevanten Subgruppen}

Aus einem unveröffentlichten systematischen Literaturreview zur Kosteneffektivität von DES der Arbeitsgruppe wurden alle Studien ausgewählt, die Subgruppenanalysen zur Kosteneffektivität enthielten. (Die Recherche zum ursprünglichen Review wurde in Embase, Medline sowie der CRD-Datenbank 2006 durchgeführt und nach den Methoden der evidenzbasierten Medizin selektiert). Extrahiert wurden folgende Parameter: Studiendesign, Definition der Subgruppen, das Effektmaß für die inkrementelle Kosten-EffektivitätsRatio (IKER), die Differenz der Effektivität zwischen DES und BMS und die IKER. Die angegebenen Kosten wurden aus der Originalwährung in Euro des Jahres 2006 konvertiert. Die Umrechnung in Euro erfolgte über Bruttoinlandsprodukt-Kaufkraftparitäten (BIP KKP) des jeweiligen Jahres (OECD PPP 2006). Euroangaben aus dem Ausland wurden ebenfalls mittels KKP in „deutsche“ Euro umgerechnet. Wenn sich die Kostenangaben der Studien auf einen Zeitraum von mehreren Jahren erstreckten, wurde eine zusätzliche Inflationsbereinigung mithilfe des allgemeinen Verbraucherpreisindexes (VPI) des Statistischen Bundesamtes durchgeführt. Anhand dieser und der im systematischen Review bereits extrahierten Daten zu Studiendesign und Studienqualität wur- 
5 Entwicklung einer Kostensensiblen Leitlinie zu Medikamente-freisetzenden Stents bei Patienten mit einer koronaren Herzkrankheit

den das methodische Vorgehen, die Ergebnisse und die Limitationen bei der Ermittlung der Kosteneffektivität von Subgruppen beschrieben.

\subsubsection{Darstellung der „Guidance“ des NICE zu DES (2007) als KSLL}

Als Beispiel einer existierenden KSLL wurde die NICE-Guidance für die Befragung von Entscheidungsträgern im Gesundheitswesen und Patienten aufbereitet (s. Kap. 9 u. 10). Hier wurden Methodik und Ergebnis beschrieben und die Kostenangaben wie oben angegeben in Euro konvertiert.

\subsection{Ergebnisse}

\subsubsection{Ergebnisse der Literaturrecherche und -selektion zu klinischen Leitlinien}

Die manuelle Recherche in Leitliniendatenbanken und auf den Internetseiten verschiedener Fachgesellschaften ergab 16o Treffer, die Suche in Medline 54. Davon wurden 208 Treffer ausgeschlossen (Duplikate, anderes Thema, keine Leitlinie, noch nicht veröffentlicht, veraltet, kein direkter Evidenzbezug). Die übrigen 6 (Silber et al. 2005, 2007; NICE 2003; King et al. 2007; Smith et al. 2005; Hodgson et al. 2003) wurden mit DELBI bewertet. Die erzielten Scores waren in etwa vergleichbar. Ausgewählt wurde das „Positionspapier der Deutschen Gesellschaft für Kardiologie zur Wirksamkeit und Sicherheit von Medikamente-freisetzenden Koronarstents (DES)“ (Silber et al. 2007), weil es sich dabei um eine evidenzbasierte aktuell erschienene deutsche Leitlinie handelte. 2008 erschien ein Update des Positionspapiers, in dem aber die Empfehlungen nicht geändert wurden. Änderungen im Update sind für den vorliegenden Zweck nicht relevant, deshalb wurde diese Publikation hier nicht berücksichtigt.

\subsubsection{Ergebnisse der Literaturrecherche und -selektion zu Studien zur Kosten- effektivität von DES bei Subgruppen}

In der Literaturrecherche für den unveröffentlichten Review gesundheitsökonomischer Studien zu DES waren 2004 und im Juli 2006 in den bibliographischen Datenbanken Embase und Medline sowie mittels Handsuche (Internet, Zeitschriften, Referenzen von Health Technology Assessment-Berichten und Leitlinien) insgesamt nach Bereinigung der Duplikate 2404 Treffer identifiziert worden. Nach dem Screening von Titeln und Abstracts anhand von vorabdefinierten Ein- und Ausschlusskriterien für Studienpopulation, Intervention und Vergleichsinterventionen, Zielgrößen und Studiendesign wurden 2359 Treffer ausgeschlossen, 5o Quellen als Volltexte bestellt und 32 Quellen zu 26 Studien in die Evidenzsynthese des systematischen Literaturreviews eingeschlossen. 12 dieser Studien (Bagust et al. 2006; Bakhai et al. 2006; Bowen et al. 2005; Cohen et al. 2004; Ekman et al. 2006; Gorenoi et al. 2005; Greenberg 
et al. 2004; Greenberg u. Cohen 2002; Hill et al. 2004; Kaiser et al. 2005; Shrive et al. 2005b; Tarricone et al. 2004) wiesen Ergebnisse zur Kosteneffektivität von Subgruppen auf und wurden deshalb zur Erstellung der KSLL oder zur Untersuchung der methodischen Limitationen in Betracht gezogen. Die Ergebnisse zur Kosteneffektivität wurden aus 6 Quellen berichtet, die für wesentliche Annahmen auch valide empirische Daten verfügbar hatten (Bagust et al. 2006; Bakhai et al. 2006; Bowen et al. 2005; Cohen et al. 2004; Hill et al. 2004; Kaiser et al. 2005; Shrive et al. 2005b). Die Ergebnisse von Bagust et al. 2006 entstammen einer aktualisierten Analyse der gleichen empirischen Daten wie in Hill et al. 2004. Nur erstere wurde berichtet, da auf dieser Analyse die NICE-Guidance zu DES aus dem Jahr 2008 beruhte.

\subsubsection{Ergebnisse aus den eingeschlossen Publikationen zur Effektivität und Kosteneffektivität von DES}

\section{Klinische Leitlinie}

Das Positionspapier der DGK zu DES ist eine evidenzbasierte Leitlinie: Es beruht auf einer systematischen Literaturrecherche und einer standardisierten Bewertung der Studienqualität. Zur Bewertung der Wirksamkeit und Sicherheit von DES im Vergleich zu BMS und untereinander wurden vorwiegend prospektive randomisierte Studien eingeschlossen, die Daten zur Wirksamkeit und Sicherheit wurden systematisch extrahiert. Insgesamt liegt umfangreiche Evidenz vor:

Es wurden 71 randomisierte Studien (RCT) zu DES mit zehn unterschiedlichen DES-Typen untersucht. Von 68 RCTs an 28.394 Patienten untersuchten 24 als primäre Zielgröße einen patientenrelevanten klinischen Endpunkt (erneute Revaskularisierung, kardialer und nichtkardialer Tod, Myokardinfarkt, Stentthrombosen). In 54 Studien wurden Patienten mit stabiler KHK in De-novo Stenosen in nativen Koronargefäßen ( $\mathrm{N}=24.207$ Patienten), in 4 Studien Patienten mit einer In-Stent-Restenose ( $\mathrm{N}=1230$ Patienten) und in $10 \mathrm{RCTs}$ Patienten mit einem ST-Streckenhebungsmyokardinfarkt ( $\mathrm{N}=2957$ Patienten) untersucht. Im Positionspapier wurden die Studienergebnisse für jede einzelne Studie in Form von Häufigkeiten der aufgetretenen Ereignisse für jeden Studienarm (DES, BMS) in Übersichtstabellen berichtet. Es wurde aber kein Effektmaß, das unmittelbar die Differenz zwischen den Interventionsgruppen quantifiziert, wie relative oder absolute Risiken, berichtet. Somit wird keine unmittelbare Aussage getroffen, um wie viele relative oder absolute Prozentpunkte DES z.B. erneute Revaskularisierungen vermindern. Die Ergebnisse wurden auch nicht für relevante Patientensubgruppen in Metaanalysen zusammengefasst. So ist ein quantitativer Vergleich der Effektivität zwischen verschiedenen Patientensubgruppen nicht möglich. 
5 Entwicklung einer Kostensensiblen Leitlinie zu Medikamente-freisetzenden Stents bei Patienten mit einer koronaren Herzkrankheit

Zur Beurteilung der Sicherheit von DES wurden hingegen bereits publizierte Metaanalysen herangezogen und getrennt für die verschiedenen DES-Typen berichtet. Es wurde insbesondere ein erhöhtes Risiko sehr später Stentthrombosen nach dem ersten Jahr nach Implantation von DES im Vergleich zu BMS sowohl für den Cypher- (statistisch signifikante Zunahme von o,6\% gegenüber BMS nach 4 Jahren) als auch für den Taxus-Stent (statistisch signifikante Zunahme von $0,4 \%$ gegenüber BMS nach 4 Jahren) nachgewiesen. Mortalität (Cypher-Stent: 6,7\% vs. BMS 5,4\%; Taxus-Stent 4,6\% vs. BMS 4,9\%) und Herzinfarktraten (Cypher-Stent: 6,4\% vs. BMS 6,2\%; Taxus-Stent 2, 8\% vs. BMS 1,8\%) waren jedoch insgesamt nicht statistisch signifikant erhöht.

Um einer erhöhten Stentthrombosegefahr zu begegnen, wurde von verschiedenen medizinischen Fachgesellschaften und Zulassungsbehörden eine Verlängerung der zusätzlichen Gabe von Clopidogrel nach der Stentimplantation über die in den Zulassungsstudien angesetzte Dauer hinaus in Betracht gezogen (King et al. 2007; Silber et al. 2005; FDA 2006). Im Positionspapier der DGK wurde festgestellt, dass für die Bestimmung der Dauer einer verlängerten antithrombotischen Medikation mit Clopidogrel zusätzlich zu Aspirin nach DES noch keine ausreichende Evidenz vorläge, und die Empfehlungen hierzu aus verschiedenen Leitlinien wurden berichtet (mindestens bis 6 Monate nach Implantation, bei besonders hohem Risiko länger). Mit der Einnahme von Clopidogrel ist wiederum ein erhöhtes Blutungsrisiko verbunden, sodass möglichst keine operativen Eingriffe erfolgen sollten. Leitlinienempfehlungen zum Einsatz von DES müssen dem Sicherheitsrisiko in der klinischen Praxis besondere Beachtung schenken, weil hier Komorbidität, Nicht-Compliance und zum Teil auch Unkenntnis bei behandelnden Ärzten eine größere Rolle spielen als unter Studienbedingungen.

In der Leitlinie werden drei Ansätze genannt, um die Indikationen neuer Technologien zu bestimmen:

1. Die Indikationen werden ausschließlich auf die Populationen mit den in den Studien geprüften Ein- und Ausschlusskriterien beschränkt;

2. man beschränkt sich auf die zugelassenen Indikationen; und

3. man berücksichtigt Kosten-Nutzen-Analysen.

Vor- und Nachteile von Ansatz 1 und 2 werden abgewogen, da im Fall von DES genau die Patienten mit dem größten Restenoserisiko den größten Nutzen haben, für verschiedene Subgruppen mit erhöhtem Risiko die Evidenzlage unterhalb des Niveaus randomisierter kontrollierter Studien liegt und diese Gruppen auch das größte Risiko für Stentthrombosen haben.

Kosten-Nutzen-Aspekte sollen laut den Autoren des Positionspapiers grundsätzlich in die Leitlinienempfehlungen einbezogen werden, Evidenz zur Kosteneffektivität wird jedoch nur anekdotisch berichtet. Zur Orientierung, bis zu welchem Betrag Interventionen als kosteneffektiv betrachtet werden können, wird in Ermangelung eines Schwellenwert im deutschen Gesundheits- 
system auf den Schwellenwert des NICE von 30.000 £ pro gewonnenem QALY (Quality adjusted life years) verwiesen. Weil die Einschränkung des Einsatzes von DES bei der Empfehlung von NICE von 2003 auch auf einer Kosteneffektivitätsanalyse beruhte und mit der größten Subgruppe in der Empfehlung des Positionspapiers übereinstimmt, geht man im Positionspapier der DGK davon aus, dass damit auch die Kosten-Nutzen-Aspekte in die Empfehlungen integriert seien.

Die Empfehlungen zum Einsatz von DES im DGK-Positionspapier sind in Tabelle 21 aufgelistet.

\section{Die klinische Leitlinie als Basis für KSLL}

In der Erstellung von KSLL sollen im ersten Schritt die Patientengruppen identifiziert werden, die den größten zusätzlichen Nutzen gegenüber dem bisherigen Verfahren aus einer medizinischen Maßnahme ziehen können bzw. diejenigen, bei denen der zusätzliche Nutzen sehr gering ist, sodass im Falle einer Rationierung nur marginaler Nutzen vorenthalten wird. Dazu muss bereits die klinische Leitlinie Angaben zur Größe des Effekts in verschiedenen

\section{Tab. 21 Empfehlungen zum Einsatz von DES im Positionspapier der DGK 2007 (nach Tab. 17 in Silber et al. 2007)}

1. Einsatz von DES bevorzugt bei erhöhtem Risiko einer Restenose

\begin{tabular}{l} 
a. stabile KHK mit zu einer Symptomatik/Myokardischämie führenden De-novo-Koronarstenose mit \\
einem Gefäßdurchmesser $\leq 3,0 \mathrm{~mm}$ und/oder einer Stenosenlänge $\geq 15 \mathrm{~mm}$ \\
\hline b. nach erfolgreicher Wiedereröffnung eines chronisch verschlossenen Koronargefäßes \\
\hline c. In-Stent-Restenose eines unbeschichteten Koronarstents \\
2. Einsatz von DES zurückhaltend bei erhöhtem Risiko einer Stentthrombose, insbesondere bei:
\end{tabular}

a. deutlich eingeschränkter LV-Funktion ( $E F<30 \%)$

b. Niereninsuffizienz

c. diffuser KHK mit Mehrgefäß-PCI

3. eher keine DES, wenn die Möglichkeit einer bzw. Compliance zur verlängerten Clopidogrelgabe nicht gegeben oder nicht eruierbar ist

a. Anamnese hinsichtlich zu erwartender Compliance schwierig zu erheben

b. multimorbide Patienten mit hoher Tablettenanzahl

c. demnächst geplante Operation

d. erhöhtes, nicht zu beseitigendes Blutungsrisiko

e. bekannte ASS-Unverträglichkeit oder Clopidogrelallergie

f. bei strikter Indikation zur Dauerantikoagulation (in Abhängigkeit vom Einzelfall)

ASS: Acetylsalicylsäure, Aspirin; EF: Ejektionsfraktion; KHK: koronare Herzkrankheit; LV: linksventrikulär; PCI: perkutane Koronarintervention 
5 Entwicklung einer Kostensensiblen Leitlinie zu Medikamente-freisetzenden Stents bei Patienten mit einer koronaren Herzkrankheit

Subgruppen machen. Zusätzlich muss auch angegeben werden, mit welcher Unsicherheit diese Effektschätzungen behaftet sind. Relevant für eine Berücksichtigung der Kosteneffektivität in Leitlinien ist auch, dass die Wirksamkeit und Sicherheit unter Praxisbedingungen abgeschätzt werden kann.

Im zweiten Schritt wird das Kosten-Effektivitätsverhältnis der verschiedenen Subgruppen herangezogen, um Subgruppen mit einem zu ungünstigen Kosten-Effektivitätsverhältnis auszuschließen.

\section{Geeignete Darstellung von Effektivitäts- und Sicherheitsdaten}

Im vorliegenden Positionspapier der DGK wurden die Ergebnisse zur Wirksamkeit von DES nicht so präsentiert, wie dies für die Erstellung einer KSLL erforderlich wäre. Es sollten Effektschätzer zu relativer und absoluter Wirksamkeit angegeben werden: Um welchen Faktor verringert sich das Risiko für patientenrelevante Ereignisse (Relative Risiken, Odds Ratios, Hazard Ratios)? Wie viele kardiovaskuläre Ereignisse werden vermieden (Risikodifferenzen)? Wenn mehrere Studien vorhanden sind, muss aus den Effektschätzern aller Einzelstudien ein gemeinsamer Effektschätzer berechnet werden (Metaanalyse), möglichst getrennt für relevante Subgruppen. Voraussetzung hierfür ist, dass die Studien hinsichtlich Studienpopulation und anderer Aspekte des Studienprotokolls nicht zu heterogen sind, sodass eine Übertragbarkeit des gemeinsamen Effektschätzers auf die Zielpopulation sinnvoll erscheint.

\section{Verfügbarkeit und Ergebnisse von Subgruppenanalysen}

Die Einzelergebnisse der Studien wurden im Positionspapier der DGK entsprechend der Einschlusskriterien der Studienpopulationen (De-novo Koronarstenosen, BMS-Instent-Restenosen, Myokardinfarkt) gruppiert. Auch bei geeigneter quantitativer Darstellung würde eine Subgruppenanalyse relativ schnell an Grenzen stoßen: Zum einen entsprechen die Einschlusskriterien für die Studienpopulationen nicht direkt den Risikofaktoren für Restenosen (z.B. lange Gefäße, kleine Gefäßdurchmesser, Diabetes, Bypassgefäße, restenotische Gefäße), die hinsichtlich des unterschiedlichen Zusatznutzens durch DES betrachtet werden müssten. Es müssten deshalb Daten zu den Subgruppen in den einzelnen RCTs vorliegen. Dazu sind größere Fallzahlen und eine entsprechende Stratifizierung bei der Randomisierung erforderlich. Die Analyse der Subgruppen mit kleinen Gefäßen und langen Läsionen basierte zum Zeitpunkt der Leitlinienerstellung auf Subgruppenanalysen von zwei größeren RCT (ca. 1050 bzw. 1300 Patienten). In der SIRIUS-Studie wurde die Randomisierung nach dem Diabetes-Status stratifiziert (Moses et al. 2003), in derTAXUS-Studie nach Diabetes-Status und Gefäßdurchmesser $(<3.0 \mathrm{~mm}$ oder $\geq 3.0 \mathrm{~mm}$ ) (Stone et al. 2004). In beiden RCT ist die relative Risikoreduktion für eine erneute Revaskularisierung über alle Subgruppen hinweg in etwa gleich zwischen 70 und $80 \%$. Dies bedeutet gleichzeitig, dass Patientengruppen mit höherem Risiko 
eine höhere absolute Risikoreduktion mit DES erwarten können, als Patienten mit niedrigerem Risiko für eine Restenose (s. Tab. 23).

\section{Übertragbarkeit der Wirksamkeit und Sicherheit in klinischen Studien auf die Popula- tion in der klinischen Praxis}

Die klinische Leitlinie befasste sich vor allem unter dem Sicherheitsaspekt (Stentthrombosen, Myokardinfarkte, Mortalität) mit den möglichen Unterschieden zwischen den Ergebnissen in den randomisierten Studien im Vergleich zum klinischen Alltag. Gerade weil erwartet wurde, dass Patienten mit erhöhtem Restenoserisiko den größten Nutzen haben, für die zum Teil noch keine Evidenz aus RCT zur Verfügung stand, ist in der klinischen Praxis der Off-label-Use, d.h. eine Anwendung von DES außerhalb der Indikationsangaben bei der Zulassung, verbreitet (6o\% in USA). Für diese Patienten reichte die Datenlage zur Sicherheit jedoch nicht aus (Farb u. Boam 2007).

Im Positionspapier der DGK wurden zur Frage der Sicherheit im klinischen Alltag zum Teil Registerdaten herangezogen. Sie entsprechen in der Regel besser der Zusammensetzung der Patienten in der klinischen Praxis. Es muss bei diesen Daten jedoch stets mit einer systematischen Verzerrung der Ergebnisse durch Risikounterschiede zwischen den Gruppen bereits zu Beginn gerechnet werden. Denn sobald eine Zuweisung einer Behandlung nicht randomisiert erfolgt, wählen Ärzte in der Regel die Behandlungsoption entsprechend der Risikoeinschätzung. Zum Teil waren in den Registern Stentthromboseraten, Myokardinfarkte und Mortalität für DES erhöht, zum Teil nicht, sodass hierdurch keine weitere Klärung erfolgte.

Einen weiteren Aspekt des klinischen Alltags, der durch die RCT nicht abgebildet wurde und der auch kostenrelevant ist, stellt der unterschiedliche Gebrauch von Clopidogrel bei BMS und DES dar. In der Praxis wird nach der Implantation von BMS zur Prävention von Stentthrombosen für einen Monat Clopidogrel verordnet. Für DES waren bereits vor dem gehäuften Auftreten von Stentthrombosen entsprechend der Zulassung 3 Monate bzw. 6 Monate Clopidogrel verordnet worden. Ein Unterschied zwischen den Gruppen durch damit assoziierte Folgekomplikationen (Blutungen) konnte in den RCT jedoch nicht festgestellt werden, weil zur Aufrechterhaltung der Verblindung die BMS-Cruppe ebenfalls länger Clopidogrel erhielt. Inzwischen wurde in den Empfehlungen verschiedener Fachgesellschaften die Dauer der Clopidogrelmedikation verlängert. Das bedeutet, dass in den klinischen Studien tendenziell unerwünschte Nebeneffekte und Kosten unterschätzt werden. In einer KSLL müssten diese Aspekte berücksichtigt werden.

Auch für die Effektivität kann nicht automatisch davon ausgegangen werden, dass die Ergebnisse aus den RCT direkt auf die Patienten im klinischen Alltag übertragbar sind. Im vorliegenden Fall gibt es zwei Faktoren, die die Übertragbarkeit einschränken können. Erstens wurden in den meisten RCT im Studien- 
5 Entwicklung einer Kostensensiblen Leitlinie zu Medikamente-freisetzenden Stents bei Patienten mit einer koronaren Herzkrankheit

protokoll Kontrollangiografien angeordnet, die in der klinischen Praxis nicht üblich sind und die aufgrund der dadurch zusätzlich ausgelösten Revaskularisierungen zu einer Überschätzung der Effektivität führen (Ruygrok et al. 1999). Zweitens ist nicht davon auszugehen, dass das durchschnittliche Restenoserisiko in den RCT mit dem Risiko in der Population in der klinischen Praxis übereinstimmt. Unterschiedliche Revaskularisierungsraten der BMSGruppe in den RCT machen das deutlich. Hierauf geht das Positionspapier nicht näher ein, zumal es stets um die über die Subgruppen hinweg etwa gleich große relative Effektivität der DES geht, d.h. um welchen Faktor verringern DES die Revaskularisierungsraten und nicht um die absoluten Risikodifferenzen zwischen Interventions- und Kontrollgruppe. Für die Krankheitslast und die ökonomische Analyse ist jedoch die absolute Anzahl an vermiedenen Ereignissen relevant. Sie hängt entscheidend vom Basisrisiko ab. Dementsprechend sollten für die Zielpopulation repräsentative PCI-Register oder Versorgungsforschungsstudien herangezogen werden, um das Restenoserisiko mit BMS in der Zielpopulation festzustellen und mit den Daten aus den RCT zu vergleichen. Denn wenn das Basisrisiko gering ist, werden auch bei starker Risikoreduktion durch die Intervention nur vergleichsweise wenige Fälle vermieden. Deshalb ist das Restenoserisiko der Zielpopulation relevant für KSLL, und die Ergebnisse aus RCT müssen für KSLL entsprechend angepasst werden.

Hier ist auch festzuhalten, dass die Häufigkeit von erneuten Revaskularisierungen zwischen verschiedenen Ländern nicht nur durch die unterschiedliche Epidemiologie der KHK beeinflusst werden kann, sondern auch durch eine unterschiedliche Versorgungspraxis. So gibt es große Unterschiede bei der Anzahl der PCI pro Einwohner, die nicht durch epidemiologische Unterschiede erklärt werden können. In Deutschland betrug die Häufigkeit 2006 ca. 3500 pro Mio. Einwohner, in Österreich und der Schweiz hingegen nur 2300 pro Mio. Einwohner (Bruckenberger 2007). Dies bedeutet, dass das Basisrisiko für eine Revaskularisierung entsprechend der Landesgepflogenheiten unterschiedlich sein kann. Bei landesspezifischen gesundheitsökonomischen Analysen kann dies von Bedeutung sein.

\section{Kosteneffektivitätsstudien}

Die 12 identifizierten Studien mit Angaben zur Kosteneffektivität in verschiedenen Subgruppen (s. Tab. 22) verfolgten im Wesentlichen vier verschiedene Ansätze, um die Kosteneffektivität zu bestimmen.

1. In DES-RCT wurden klinische Effektdaten, der Ressourcenverbrauch und die Preise direkt erhoben und daraus wurde die Kosteneffektivität berechnet (Bakhai et al. 2006; Cohen et al. 2004; Kaiser et al. 2005).

2. In DES-RCT wurden klinische Effektdaten erhoben. Sie wurden in einem Modell mit länderspezifischem Ressourcenverbrauch und länderspezifischen Preisen kombiniert (Ekman et al. 2006). 
Tab. 22 Studiendesign der eingeschlossenen gesundheitsökonomischen Studien

\begin{tabular}{|c|c|c|c|c|c|c|c|c|c|}
\hline Autor & Jahr & Land & $\begin{array}{l}\text { Sponsor } \\
\text { Industrie }\end{array}$ & $\begin{array}{c}\text { Inter- } \\
\text { vention }\end{array}$ & Vergleich & $\begin{array}{l}\text { Effekt- } \\
\text { maße }\end{array}$ & $\begin{array}{l}\text { Studien- } \\
\text { typ }\end{array}$ & $\begin{array}{l}\text { Berechnungs- } \\
\text { ansatz KE }\end{array}$ & $\begin{array}{l}\text { Perspektive/ } \\
\text { Zeithorizont }\end{array}$ \\
\hline Bagust et al. & 2006 & GB & nein & $\begin{array}{c}\text { DES } \\
\text { (unspez.) }\end{array}$ & $\begin{array}{c}\text { BMS } \\
\text { (unspez.) }\end{array}$ & QALY & $\begin{array}{l}\text { KEA, KNA } \\
\text { Modell }\end{array}$ & $\begin{array}{c}3 \\
\text { siehe Text }\end{array}$ & $\begin{array}{c}\text { Kostenträger } \\
1 \text { Jahr }\end{array}$ \\
\hline Bakhai et al. & 2006 & USA & $\begin{array}{l}\text { Boston } \\
\text { Sc. }\end{array}$ & $\begin{array}{l}\text { Taxus } \\
\text { (PES) }\end{array}$ & $\begin{array}{c}\text { BMS } \\
\text { baugleich }\end{array}$ & $\begin{array}{l}\text { TVR, } \\
\text { QALY }\end{array}$ & $\begin{array}{c}\text { KEA, KNA } \\
\text { RCT }\end{array}$ & $\begin{array}{c}1 \\
\text { siehe Text }\end{array}$ & $\begin{array}{c}\text { gesellschaftlich } \\
1 \text { Jahr }\end{array}$ \\
\hline Bowen et al. & 2005 & Kanada & nein & $\begin{array}{l}\text { DES } \\
\text { (Cypher } \\
\text { und } \\
\text { Taxus) }\end{array}$ & $\begin{array}{c}\text { BMS } \\
\text { (unspez.) }\end{array}$ & $\begin{array}{c}\text { Revask, } \\
\text { QALY }\end{array}$ & $\begin{array}{c}\text { KEA, KNA } \\
\text { Modell }\end{array}$ & $\begin{array}{c}4 \\
\text { siehe Text }\end{array}$ & $\begin{array}{c}\text { Kostenträger } \\
1 \text { Jahr }\end{array}$ \\
\hline Cohen et al. & 2004 & USA & Cordis & Cypher & $\begin{array}{c}\text { BMS } \\
\text { baugleich }\end{array}$ & $\begin{array}{c}\text { alle } \\
\text { Revask, } \\
\text { QALY }\end{array}$ & $\begin{array}{c}\text { KEA, KNA } \\
\text { RCT }\end{array}$ & $\begin{array}{c}1 \\
\text { siehe Text }\end{array}$ & $\begin{array}{c}\text { gesellschaftlich } \\
1 \text { Jahr }\end{array}$ \\
\hline Ekman et al. & 2006 & $\begin{array}{l}\text { Schwe- } \\
\text { den }\end{array}$ & $\begin{array}{l}\text { Boston } \\
\text { Sc. }\end{array}$ & Taxus & $\begin{array}{c}\text { BMS } \\
\text { (unspez.) }\end{array}$ & $\begin{array}{l}\text { TLR, } \\
\text { QALY }\end{array}$ & $\begin{array}{c}\text { KEA, KNA } \\
\text { Modell }\end{array}$ & $\begin{array}{c}2 \\
\text { siehe Text }\end{array}$ & $\begin{array}{c}\text { Kostenträger } 1 \\
\text { und } 2 \text { lahre }\end{array}$ \\
\hline $\begin{array}{l}\text { Greenberg u. } \\
\text { Cohen }\end{array}$ & 2002 & USA & nein & $\begin{array}{c}\text { DES } \\
\text { (unspez.) }\end{array}$ & $\begin{array}{c}\text { BMS } \\
\text { (unspez.) }\end{array}$ & Revask & $\begin{array}{c}\text { KEA } \\
\text { Modell }\end{array}$ & $\begin{array}{c}3 \\
\text { siehe Text }\end{array}$ & $\begin{array}{c}\text { Kostenträger } \\
1 \text { Jahr }\end{array}$ \\
\hline $\begin{array}{l}\text { Greenberg et } \\
\text { al. }\end{array}$ & 2004 & USA & nein & $\begin{array}{c}\text { DES } \\
\text { (Cypher) }\end{array}$ & $\begin{array}{c}\text { BMS } \\
\text { (unspez.) }\end{array}$ & TVR & $\begin{array}{c}\text { KEA } \\
\text { Modell }\end{array}$ & $\begin{array}{c}3 \\
\text { siehe Text }\end{array}$ & $\begin{array}{l}\text { Gesundheits- } \\
\text { system/2 Jahre }\end{array}$ \\
\hline Hill et al. & 2004 & GB & nein & $\begin{array}{c}\text { DES } \\
\text { (unspez.) }\end{array}$ & $\begin{array}{c}\text { BMS } \\
\text { (unspez.) }\end{array}$ & $\begin{array}{c}\text { Revask, } \\
\text { QALY }\end{array}$ & $\begin{array}{c}\text { KNA } \\
\text { Modell }\end{array}$ & $\begin{array}{c}3 \\
\text { siehe Text }\end{array}$ & $\begin{array}{c}\text { Kostenträger } \\
1 \text { Jahr }\end{array}$ \\
\hline Kaiser et al. & 2005 & Schweiz & nein & Cypher & Taxus & $\begin{array}{l}\text { MACE, } \\
\text { QALY }\end{array}$ & $\begin{array}{c}\text { KEA, KNA } \\
\text { RCT }\end{array}$ & $\begin{array}{c}1 \\
\text { siehe Text }\end{array}$ & $\begin{array}{c}\text { Kostenträger } 6 \\
\text { Monate }\end{array}$ \\
\hline Shrive et al. & 2005 & Kanada & nein & Cypher & $\begin{array}{c}\text { BMS } \\
\text { (unspez.) }\end{array}$ & QALY & $\begin{array}{c}\text { KNA } \\
\text { Modell }\end{array}$ & $\begin{array}{c}3 \\
\text { siehe Text }\end{array}$ & $\begin{array}{l}\text { Kostenträger } \\
\text { lebenslang }\end{array}$ \\
\hline Szucs et al. & 2005 & Schweiz & nein & DES & $\begin{array}{c}\text { BMS } \\
\text { (unspez.) }\end{array}$ & TLR & $\begin{array}{c}\text { KKA } \\
\text { Modell }\end{array}$ & $\begin{array}{c}3 \\
\text { siehe Text }\end{array}$ & $\begin{array}{l}\text { Kostenträger } \\
\text { nicht genannt }\end{array}$ \\
\hline Tarricone et al. & 2004 & Italien & Cordis & $\begin{array}{l}\text { Cypher } \\
\text { (SES) }\end{array}$ & $\begin{array}{c}\text { BMS } \\
\text { (unspez.) }\end{array}$ & $\begin{array}{l}\text { TLR, } \\
\text { MACE }\end{array}$ & $\begin{array}{c}\text { KKA } \\
\text { Modell }\end{array}$ & $\begin{array}{c}3 \\
\text { siehe Text }\end{array}$ & $\begin{array}{l}\text { Kostenträger } \\
1 \text { Jahr }\end{array}$ \\
\hline
\end{tabular}

BMS = Metallstent, DES = Medikamente-freisetzender Stent, KEA = Kosten-Effektivitätsanalyse, KNA = Kosten-Nutzwertanalyse, KKA = Kosten-Konsequenzenanalyse, MACE = Große kardiale Ereignisse (kardialer Tod, MI, TVR oder TLR), $\mathrm{MI}=$ Myokardinfarkt, $\mathrm{QALY}=$ Qualitätsadjustierte Lebensjahre, Revask = Revaskularisierungen, $\mathrm{TLR}=$ Zielläsionrevaskularisierung, TVR = Zielgefäßrevaskularisierung

3. Ereignisraten von BMS, die als repräsentativ für die Zielpopulation betrachtet wurden (Register, BMS-RCT, Casemix aus Kliniken), wurden mithilfe eines Modells mit der relativen Effektivität von DES aus Metaanalysen von DES-RCT und dem landesspezifischen Ressourcenverbrauch sowie länderspezifischen Preisen kombiniert (Bagust et al. 2006; Greenberg u. Cohen 2002; Greenberg et al. 2004; Hill et al. 2004; Shrive et al. 2005b; Szucs et al. 2005; Tarricone et al. 2004).

4. Störgrößen adjustierte Effektivitätsdaten wurden in einem DES-/BMSRegister erhoben und mit landesspezifischen Kostendaten kombiniert (Bowen et al. 2005). 
5 Entwicklung einer Kostensensiblen Leitlinie zu Medikamente-freisetzenden Stents bei Patienten mit einer koronaren Herzkrankheit

In vier Studien wurden klinische Effektmaße (Revaskularisierungen in verschiedenen Definitionen), in zwei Studien qualitätsadjustierte Lebensjahre (QALY) und in sechs Studien wurde beides verwendet (s. Tab. 22).

Für Deutschland waren bis zum Ende des Recherchezeitraums keine gesundheitsökonomischen Studien zu DES mit Subgruppenanalysen durchgeführt worden. Die methodischen Besonderheiten von gesundheitsökonomischen Studien, die der Entwicklung von KSLL dienen können, lassen sich jedoch auch an Studien aus anderen Ländern veranschaulichen.

\section{Kosteneffektivität von Subgruppen in RCTs}

Von den drei Kosteneffektivitätsstudien mit Subgruppenanalysen, die parallel mit den RCT durchgeführt wurden (Bakhai et al. 2006; Cohen et al. 2004; Kaiser et al. 2005), sind zwei aus den USA, die dritte ist aus der Schweiz. Die Schweizer Studie BASKET basiert auf einem pragmatischen RCT, d.h. es wurden keine Studienprotokoll-induzierten Angiografien durchgeführt, als Vergleichs-BMS wurde ein Stent der 3. Generation mit geringeren Restenoseraten verwendet, statt zu DES baugleichen BMS und die Studienpopulation entstammte der Alltagsversorgung in der Schweiz. Die Ergebnisse der Subgruppenanalysen sind in Tabelle 23 gezeigt. Es wurden die zusätzlichen Kosten für DES pro vermiedener Revaskularisierung angegeben.

Es ist unklar, wie groß die gesellschaftliche Zahlungsbereitschaft für vermiedene Revaskularisierungen ist. Häufig wird in kardiologischen gesundheitsökonomischen Studien in den USA 10.0oo Dollar als Schwellenwert gewählt, weil eine Reihe von Interventionen, die üblicherweise finanziert werden, unter diesem Wert liegen (Ryan u. Cohen 2006). In den beiden US-amerikanischen Kosteneffektivitätsstudien wird dieser Wert in allen Subgruppen unterschritten, außer im Fall von Gefäßen mit einem Durchmesser über $3 \mathrm{~mm}$ in derTAXUS IV-Studie. Die durchschnittlichen IKER liegen bei 4.337 Euro (TAXUS IV) bzw. 1.684 Euro (SIRIUS). Dementsprechend kommen die Autoren zum Schluss, dass DES für die Studienpopulationen von SIRIUS und TAXUS IV im US-amerikanischen Gesundheitssystem kosteneffektiv seien. Vergleicht man diese Kosteneffektivitätsanalyse der Subgruppen mit den Empfehlungen des Positionspapiers der DGK, so wäre wie auch von den Autoren des Positionspapiers postuliert, mit der Empfehlung, DES nur bei Gefäßdurchmessern unter $3 \mathrm{~mm}$ zu verwenden, der Kosteneffektivitätsaspekt bereits berücksichtigt. Deutlich ungünstigere Kosteneffektivitätsverhältnisse wurden in der BASKET-Studie berichtet. Im Durchschnitt lag die IKER bei 11.120 Euro pro vermiedener Revaskularisierung, die Subgruppen der über 65-Jährigen, Patienten mit Läsionslängen über $20 \mathrm{~mm}$, mit drei betroffenen Gefäßen, sowie Patienten mit mindestens einem Stent mit einem Durchmesser von 2,5 mm oder Patienten, bei denen mehr als ein Segment betroffen waren, lagen unter 10.0oo Euro pro vermiedener Revaskularisierung. In den beiden letztgenannten Gruppen war der Einsatz von DES dominant d.h. effektiver und billiger. 
Tab. 23 Inkrementelle Kosten-Effektivitäts-Verhältnisse (IKER) von DES im Vergleich zu BMS in RCT währungskonvertiert in EURO

\begin{tabular}{|c|c|c|c|c|c|c|c|}
\hline TAXUS IV & Revask & Euro pro & SIRIUS & Revask & Euro pro & BASKET & Euro pro \\
\hline Subgruppen & $\begin{array}{l}\triangle \mathrm{DES}- \\
\text { BMS }\end{array}$ & $\begin{array}{c}\text { vermied. } \\
\text { Revask } \\
\text { (TVR) }\end{array}$ & & $\begin{array}{l}\triangle \mathrm{DES}- \\
\text { BMS }\end{array}$ & $\begin{array}{c}\text { vermied. } \\
\text { Revask } \\
\text { (alle) }\end{array}$ & Subgruppen & $\begin{array}{c}\text { vermied. } \\
\text { Revask }\end{array}$ \\
\hline alle & $12,2 \%$ & 4.337 & alle & $19,1 \%$ & 1.684 & alle & $11.120^{*}$ \\
\hline Diabetiker & $11,7 \%$ & DOM & Diabetiker & $17,3 \%$ & 2.425 & $\leq 65$ lahre & $52.730^{*}$ \\
\hline Nicht-Diabetiker & $9,2 \%$ & 8.703 & Nicht-Diabetiker & $18,7 \%$ & 2.014 & $>65$ lahre & $7.909^{*}$ \\
\hline $\mathrm{D} \leq 2,5 \mathrm{~mm}$ & $10,4 \%$ & DOM & $\mathrm{D}<2,5 \mathrm{~mm}$ & $23,0 \%$ & DOM & D 2,5 mm o Stents & $52.730^{*}$ \\
\hline$D>2,5<3,0 \mathrm{~mm}$ & $10,5 \%$ & 4.718 & D 2,5-3,0 mm & $20,0 \%$ & 1.373 & $\begin{array}{l}\text { D } 2,5 \mathrm{~mm} \geq 1 \\
\text { Stents }\end{array}$ & DOM* \\
\hline$D \geq 3,0 \mathrm{~mm}$ & $7,8 \%$ & 23.248 & $\mathrm{D}>3,0 \mathrm{~mm}$ & $16,0 \%$ & 6.334 & & \\
\hline $\mathrm{L} \leq 20 \mathrm{~mm}$ & $8,7 \%$ & 6.212 & $\mathrm{~L}<15 \mathrm{~mm}$ & $18,1 \%$ & 4.353 & $\mathrm{~L} \leq 20 \mathrm{~mm}$ & $>52.530^{*}$ \\
\hline $\mathrm{L}>20 \mathrm{~mm}$ & $20 \%$ & 4.610 & $\mathrm{~L} 15-20 \mathrm{~mm}$ & $9,8 \%$ & 4.551 & $\mathrm{~L}>20 \mathrm{~mm}$ & $2.636^{*}$ \\
\hline LAD & $10,6 \%$ & 2.563 & $\mathrm{~L}>20 \mathrm{~mm}$ & $37,2 \%$ & DOM & 1-2 Gefäße & $>52.530^{*}$ \\
\hline \multirow[t]{4}{*}{ Non-LAD } & $9,2 \%$ & 8.109 & Pred $10-15 \%$ & $15,6 \%$ & 3.804 & 3 Gefäße & $5.273^{*}$ \\
\hline & & & Pred $15-20 \%$ & $16,2 \%$ & 5.908 & 1 Segmente & $>52.530^{*}$ \\
\hline & & & Pred $20-25 \%$ & $26,1 \%$ & 519 & > 1 Segmente & DOM* \\
\hline & & & Pred $25-30 \%$ & $21,9 \%$ & DOM & & \\
\hline
\end{tabular}

* Wert aus Graphik abgelesen

BMS = Metallstent, DES = Medikamente-freisetzender Stent, D = Durchmesser des Gefäßes oder Stents, Diabet = Diabetiker, DOM = dominant, d.h. billiger und effektiver, $L=$ Länge der Läsion, $L A D=$ Left Anterior Descending, einer der beiden Hauptäste der linken Koronararterie, Pred = Predicted Risk, Seg = Segment, TVR = Target vessel revascularisation

Die höheren IKER in BASKET beruhen vor allem auf dem geringeren Effektivitätszuwachs gegenüber BMS (3,9\% vermiedene Revaskularisierungen). Für SIRIUS und BASKET wurden auch QALY als Effektmaß berichtet. Diese beruhen jedoch nur bei BASKET auf einer Erhebung der gesundheitsbezogenen Lebensqualität unmittelbar in der Studie. Bei SIRIUS wurden die Werte aus anderen Revaskularisierungsstudien übertragen. In SIRIUS wurde ein Wert von 28.108 Euro pro gewonnenem QALY berichtet, in BASKET 77.284 bzW. 57.524 Euro je nach Erhebungsinstrument (EQ5D bzw. VAS) für die Lebensqualität.

\section{Kosteneffektivität in Subgruppen in der Alltagspraxis}

Neben der internen Validität der zugrundeliegenden Studien ist die Übertragbarkeit auf die Zielpopulation ein entscheidender Aspekt für Leitlinienempfehlungen zur Indikation für neue Technologien. Im vorliegenden Beispiel wird davon ausgegangen, dass die Zielpopulation die Patienten umfasst, die bisher eine Indikation für eine PCI mit einem BMS hatten. Grundsätzlich haben alle Modellierungsstudien zur Kosteneffektivität von DES versucht, den 
5 Entwicklung einer Kostensensiblen Leitlinie zu Medikamente-freisetzenden Stents bei Patienten mit einer koronaren Herzkrankheit

Aspekt der Übertragbarkeit zu berücksichtigen (s. Ansätze 2 bis 4 in Abschnitt „Kosteneffektivitätsstudien“). Im Folgenden werden jedoch nur die Studien näher betrachtet, für die tatsächlich auch zusätzliche valide Daten zur Verfügung standen, um die Annahmen in den Modellierungen empirisch zu stützen. In drei Studien standen Daten aus Registern mit Patienten aus der Routineversorgung zu Patientencharakteristika und der Häufigkeit erneuter Revaskularisierungen nach dem Einsatz von BMS zur Verfügung (Bagust et al. 2006; Bowen et al. 2005; Shrive et al. 2005b).

Die Subgruppen der Studien sind nur teilweise vergleichbar (s. Tab. 24 und Tab. 25). In den Modellierungen von Bagust et al. und Bowen et al. sind die IKER in den meisten Gruppen sehr hoch und überschreiten bei weitem die Grenze von 100.00o Euro/QALY, die häufig als die oberste Grenze einer kosteneffektiven Maßnahme (50.000-100.00o Dollar) betrachtet wird (Schöffski u. Schumann 2008). Nur Personen mit drei bis vier Risikofaktoren für erneute Revaskularisierungen, Patienten mit elektivem Eingriff oder mit mindestens einem Risikofaktor bei nichtelektivem Eingriff liegen unter diesem Wert, nur in der höchsten Risikogruppe sind DES dominant. Bei Bowen et al. liegt selbst

Tab. 24 Kosteneffektivität (IKER) von DES im Vergleich zu BMS mit Patientencharakteristika aus PCI-Registern, Bagust et al. 2006 und Shrive et al. 2005b

\begin{tabular}{|c|c|c|c|c|c|}
\hline $\begin{array}{l}\text { Bagust et al. } \\
2006\end{array}$ & Cypher-Stent & Taxus-Stent & $\begin{array}{l}\text { Shrive et al. } \\
\text { 2005b }\end{array}$ & QALY & DES \\
\hline Subgruppen & $\begin{array}{c}\text { Euro pro } \\
\text { gewonnenem QALY }\end{array}$ & $\begin{array}{c}\text { Euro pro } \\
\text { gewonnenem QALY }\end{array}$ & Subgruppen & $\begin{array}{l}\triangle D E S- \\
\text { BMS }\end{array}$ & $\begin{array}{c}\text { Euro pro } \\
\text { gewonnenem QALY }\end{array}$ \\
\hline alle & - & - & alle & 0,04 & 48.768 \\
\hline $\begin{array}{l}\text { elektiv, kein RF, } \\
\text { Risiko 5,6\%* }\end{array}$ & 365.135 & 495.813 & $<65$ lahre & 0,04 & 60.181 \\
\hline $\begin{array}{l}\text { elektiv, } 1 \text { RF, } \\
\text { Risiko 8,4\%* }\end{array}$ & 274.654 & 381.642 & $65-75$ Jahre & 0,05 & 39.400 \\
\hline $\begin{array}{l}\text { elektiv, } 2 \text { RF, } \\
\text { Risiko 16,6\%* }\end{array}$ & 130.220 & 198.998 & $>75$ lahre & 0,07 & 33.327 \\
\hline $\begin{array}{l}\text { elektiv, } 3 \text { oder } 4 \\
\text { RF, Risiko 24,6\%* }\end{array}$ & 78.865 & 134.346 & Nicht-Diabetiker & 0,04 & 52.639 \\
\hline $\begin{array}{l}\text { nonelektiv, kein } \\
\text { RF, Risiko 9,9\%* }\end{array}$ & 204.194 & 299.261 & Diabetiker & 0,06 & 36.654 \\
\hline $\begin{array}{l}\text { nonelektiv, } 1 \text { RF, } \\
\text { Risiko 22,2\%* }\end{array}$ & 46.769 & 100.416 & & & \\
\hline $\begin{array}{l}\text { nonelektiv, } 2 \text { RF, } \\
\text { Risiko 40,4\%* }\end{array}$ & DOM & DOM & & & \\
\hline
\end{tabular}

*Risikofaktoren für elektive Patienten waren Kalzifizierung, Angulation $>45^{\circ}$, Instent-Restenose, 3 betroffene Gefäße, für nichtelektive Patienten waren es Gefäßdurchmesser < 2 mm oder frühere Bypassoperation. Die Risikoangabe bezieht sich auf eine erneute Revaskularisierung nach der Implantation von BMS

BMS = Metallstent, DES = Medikamente-freisetzender Stent, Diabet = Diabetiker, DOM = dominant, d.h. billiger und effektiver, $\mathrm{L}=$ Länge der Läsion, $\mathrm{QALY}=$ qualitätsadjustiertes Lebensjahr. Die Nutzwerte zur Berechnung der QALY wurden nur bei Shrive et al. empirisch erhoben, bei Bagust et al. wurden sie einer Studie an Patienten mit Mehrgefäßerkrankung entnommen. 
Tab. 25 Kosteneffektivität (IKER) von DES im Vergleich zu BMS mit Patientencharakteristika aus PCI-Registern, Bowen et al. 2005

\begin{tabular}{|c|c|c|c|c|c|c|c|c|}
\hline \multirow{2}{*}{$\begin{array}{l}\text { Bowen } \\
\text { et al. } 2005 \\
\text { Subgruppen }\end{array}$} & \multicolumn{2}{|c|}{$\begin{array}{l}\text { Diabetiker } \\
\text { ohne MI }\end{array}$} & \multicolumn{2}{|c|}{$\begin{array}{l}\text { Diabetiker } \\
\text { mit MI }\end{array}$} & \multicolumn{2}{|c|}{$\begin{array}{l}\text { Nicht-Diabetiker } \\
\text { ohne MI }\end{array}$} & \multicolumn{2}{|c|}{$\begin{array}{c}\text { Nicht-Diabetiker } \\
\text { mit MI }\end{array}$} \\
\hline & $\begin{array}{c}\text { Euro pro } \\
\text { vermied. } \\
\text { Revask }\end{array}$ & $\begin{array}{l}\text { Euro } \\
\text { gew. } \\
\text { QALY }\end{array}$ & $\begin{array}{c}\text { Euro pro } \\
\text { vermied. } \\
\text { Revask }\end{array}$ & $\begin{array}{l}\text { Euro } \\
\text { gew. } \\
\text { QALY }\end{array}$ & $\begin{array}{c}\text { Euro pro } \\
\text { vermied. } \\
\text { Revask }\end{array}$ & $\begin{array}{l}\text { Euro } \\
\text { gew. } \\
\text { QALY }\end{array}$ & $\begin{array}{c}\text { Euro pro } \\
\text { vermied. } \\
\text { Revask }\end{array}$ & $\begin{array}{l}\text { Euro } \\
\text { gew. } \\
\text { QALY }\end{array}$ \\
\hline alle & 36.723 & 842.968 & 13.184 & 326.352 & 71.002 & 1.653 .807 & 51.881 & 1.257.117 \\
\hline $\begin{array}{l}\mathrm{L}>20 \mathrm{~mm} \text { und } \\
\mathrm{D}<2,75 \mathrm{~mm}\end{array}$ & 7.212 & 166.431 & - & - & 32.764 & 726.150 & 11.642 & 293.233 \\
\hline $\mathrm{L}>20 \mathrm{~mm}$ & 9.437 & 217.461 & - & - & 31.765 & 735.486 & 22.053 & 524.982 \\
\hline $\mathrm{L} \leq 20 \mathrm{~mm}$ & 83.111 & 1.899 .924 & - & - & 115.472 & 2.693 .676 & 193.434 & 4.731 .498 \\
\hline $\mathrm{D}<2,75 \mathrm{~mm}$ & 21.018 & 482.522 & - & - & 32.564 & 751.674 & 3.205 .497 & 79.089 .022 \\
\hline $\mathrm{D} \geq 2,75 \mathrm{~mm}$ & 49.547 & 1.135 .926 & - & - & 120.061 & 2.805 .429 & 38.728 & 933.249 \\
\hline $\begin{array}{l}\mathrm{L}>20 \mathrm{~mm} \\
\text { oder D } \\
<2,75 \mathrm{~mm}\end{array}$ & 15.474 & 355.624 & - & - & 32.630 & 760.180 & 49.301 & 1.180 .797 \\
\hline $\begin{array}{l}\mathrm{L} \leq 20 \mathrm{~mm} \text { und } \\
\mathrm{D} \geq 2,75 \mathrm{~mm}\end{array}$ & 263.473 & 6.022 .969 & - & - & BMS DOM & BMS DOM & 63.443 & 1.554 .221 \\
\hline
\end{tabular}

BMS = Metallstent, DES = Medikamente-freisetzender Stent, D = Durchmesser des Gefäßes, Diabet = Diabetiker, DOM = dominant, d.h. billiger und effektiver, $L$ = Länge der Läsion, $Q A L Y=$ qualitätsadjustiertes Lebensjahr

Nutzwerte zur Berechnung von QALY nicht direkt erhoben, sondern aus der ARTS-Studie

die Gruppe mit dem höchsten Risiko (Diabetiker mit langen Läsionen und kleinen Gefäßen) bei einem Wert von 166.431 Euro/QALY. Wird hingegen der Wert von 10.00o Euro/vermiedener Revaskularisierung verwendet, so liegen Diabetiker mit langen Läsionen und kleinen Gefäßen und Diabetiker mit langen Läsionen darunter, alle anderen Gruppen darüber. Die Studie von Shrive berichtet hingegen sehr viel niedrigere IKER von maximal 6o.181 Euro/QALY bei Patienten unter 65 Jahren. Alle anderen Werte liegen darunter und würden bei einer Zahlungsbereitschaft von ca. 52.0oo Euro als kosteneffektiv betrachtet. Der große Unterschied zwischen den Ergebnissen von Shrive und den beiden anderen Studien liegt vor allem im unterschiedlichen Zugewinn an QALY begründet. Bei Bowen et al. liegt dieser in der Gruppe mit dem höchsten Nutzen bei o,oo63, bei Shrive mit o,o7 beim Zehnfachen dieses Wertes .

\section{Heterogenität der Studienergebnisse}

Insgesamt ist festzustellen, dass die Studien, die die Revaskularisierungsraten aus Routineregistern entnehmen, zu deutlich ungünstigeren IKER kommen und dass die Unterschiede zwischen verschiedenen Studien sehr groß sind. Neben den Annahmen zu Charakteristika der PCI-Population(Revaskularisierungsraten, Anzahl der verwendeten Stents) unterscheiden sich auch die Kosten in den in die Kostenberechnung einbezogenen Komponenten, den Preisen 
5 Entwicklung einer Kostensensiblen Leitlinie zu Medikamente-freisetzenden Stents bei Patienten mit einer koronaren Herzkrankheit

für DES und BMS sowie für die Prozeduren, die ambulante Versorgung und relevante Arzneimittel, je nachdem in welchem Land und zu welchem Zeitpunkt die jeweilige Studie durchgeführt wurde (s. Tab. 26). Dementsprechend sind auch die Ergebnisse für die IKER sehr unterschiedlich.

\section{Relevanz für KSLL}

Eine unmittelbare Empfehlung für deutsche KSLL lässt sich aus diesen Studien nicht ableiten. Zum einen sind die verwendeten Subgruppen nicht kompatibel mit den Subgruppen aus den klinischen Leitlinien. Desweiteren erlauben systematische Übersichtsarbeiten zu gesundheitsökonomischen Evaluationen

Tab. 26 Kostenkomponenten der Studien, deren IKER in den Tabellen 23-25 berichtet wurden

\begin{tabular}{|c|c|c|c|c|c|c|c|c|c|c|}
\hline Studie & $\begin{array}{l}\text { Bezugs- } \\
\text { jahr }\end{array}$ & $\begin{array}{l}\text { berücksichtigte } \\
\text { Ressourcenver- } \\
\text { bräuche }\end{array}$ & $\begin{array}{c}\text { Stents } \\
n\end{array}$ & $\begin{array}{l}\text { Kosten/ } \\
\text { Stent } \\
\text { DES }\end{array}$ & $\begin{array}{c}\text { Kosten/ } \\
\text { Stent } \\
\text { BMS }\end{array}$ & $\begin{array}{l}\text { Diff } \\
\text { DES- } \\
\text { BMS- } \\
\text { Stent }\end{array}$ & $\begin{array}{l}\text { Kosten } \\
\text { initial } \\
\text { DES }\end{array}$ & $\begin{array}{c}\text { Kosten } \\
\text { initial } \\
\text { BMS }\end{array}$ & $\begin{array}{c}\text { Kosten FU } \\
1 \text { Jahr } \\
\text { DES }\end{array}$ & $\begin{array}{c}\text { Kosten FU } \\
1 \text { Jahr } \\
\text { BMS }\end{array}$ \\
\hline $\begin{array}{l}\text { Bagust } \\
\text { et al. }\end{array}$ & 2003 & $\begin{array}{l}\text { direkt: initiale } \\
\text { Prozedur, FU: } \\
\text { Klinik, } \\
\text { ambulant }\end{array}$ & 1,9 & 1.330 & 566 & 764 & - & - & - & - \\
\hline $\begin{array}{l}\text { Bakhai } \\
\text { et al. }\end{array}$ & 2004 & $\begin{array}{l}\text { direkt: initiale } \\
\text { Prozedur, FU: } \\
\text { Klinik, } \\
\text { Clopidogrel }\end{array}$ & 1,3 & 2.503 & 742 & 1.761 & 10.287 & 8.406 & 3.233 & 4.584 \\
\hline $\begin{array}{l}\text { Bowen } \\
\text { et al. }\end{array}$ & $\begin{array}{c}2003 / \\
2004\end{array}$ & $\begin{array}{l}\text { direkt: initiale } \\
\text { Prozedur (nur } \\
\text { Stents), FU: PCI } \\
\text { mit/ohne Stent, } \\
\text { CABG }\end{array}$ & 1,5 & 1.414 & 447 & 967 & 2.092 & 661 & $\begin{array}{c}- \\
417^{*}\end{array}$ & $\begin{array}{c}- \\
558^{*}\end{array}$ \\
\hline $\begin{array}{l}\text { Cohen } \\
\text { et al. }\end{array}$ & 2002 & $\begin{array}{l}\text { direkt: initiale } \\
\text { Prozedur, FU: } \\
\text { Klinik, } \\
\text { ambulant, } \\
\text { Clopidogrel }\end{array}$ & 1,4 & 2.960 & 919 & 2.041 & 11.579 & 8.639 & 5.581 & 8.206 \\
\hline $\begin{array}{l}\text { Kaiser } \\
\text { et al. }\end{array}$ & - & $\begin{array}{l}\text { direkt: initiale } \\
\text { Prozedur, FU: } \\
\text { Klinik } \\
\text { (Aufenthalt, } \\
\text { Prozeduren, } \\
\text { Stents) }\end{array}$ & 1,9 & $\begin{array}{l}\text { Cypher } \\
2.510 ; \\
2.262 \\
\text { Taxus } \\
2.041\end{array}$ & $\begin{array}{l}\text { BMS } \\
1.192 ; \\
1.329\end{array}$ & $\begin{array}{c}\text { Cypher } \\
933- \\
1.318 \\
\text { Taxus } \\
723- \\
849\end{array}$ & $\begin{array}{c}\text { DES } \\
10.199 \\
\text { Cypher } \\
10.756 \\
\text { Taxus } \\
9.620\end{array}$ & 8.916 & $\begin{array}{c}6 \text { Monate } \\
\text { DES } 921 \\
\text { Cypher } 713 \\
\text { Taxus } 1.116\end{array}$ & $\begin{array}{c}6 \text { Monate } \\
1.250\end{array}$ \\
\hline $\begin{array}{l}\text { Shrive } \\
\text { et al. }\end{array}$ & 2002 & $\begin{array}{l}\text { direkt: initiale } \\
\text { Prozedur, FU: } \\
\text { Klinik, } \\
\text { ambulant, } \\
\text { Arzneimittel, } \\
\text { Pflegekosten }\end{array}$ & 1,4 & 2.408 & 415 & 1.993 & - & - & - & - \\
\hline
\end{tabular}

*selbst berechnet 
zwar einen Einblick, welche Faktoren die Kosteneffektivität maßgeblich beeinflussen, wenn darunter jedoch nicht valide und aktuelle Studien aus dem relevanten Land und Versorgungskontext enthalten sind, sind eigene Modellrechnungen (Siebert 2005) oder unter Umständen auch eigene empirische Studien erforderlich, um aussagekräftige KSLL zu erstellen.

\section{Die „Guidance“ des National Institute for Health and Clinical Excellence zu DES als Beispiel einer KSLL}

Im Juli 2008 wurde vom NICE folgende Leitlinie zu DES herausgegeben:

Der Einsatz von DES bei der perkutanen Koronarintervention zur Behandlung der koronaren Herzkrankheit wird für die zugelassenen Indikationen nur dann empfohlen wenn

- das zu behandelnde Zielgefäß einen Durchmesser von weniger als $3 \mathrm{~mm}$ hat oder die Läsion länger als $15 \mathrm{~mm}$ ist und

- der Preisunterschied zwischen DES und konventionellen Metallstents (BMS) nicht mehr als 300 Pfund (398 Euro) beträgt.

Dies ist ein Beispiel einer KSLL. Die Empfehlung basiert auf dem oben berichteten ökonomischen Modell von Bagust et al. (2006), dem auch ein systematischer Literaturreview zur klinischen Wirksamkeit mit einer Metaanalyse aus 17 RCT vorausging. Die Berücksichtigung der Kosteneffektivität erfolgte einerseits durch die Einschränkung der Verwendung von DES auf Patienten mit einem erhöhten Risiko und durch die Beschränkung auf einen Mehrpreis von 300 Pfund für DES. Die Ermittlung des Preises beruht auf einer Schwellenwertanalyse mithilfe des ökonomischen Modells, die für alle Subgruppen den Mehrpreis ermittelte, wann der Einsatz kosteneffektiv (30.0oo Pfund/QALY) oder kostenneutral ist (o Pfund/QALY). Die in der Leitlinie gewählte Risikokategorie (s. Tab. 27) stimmt nicht mit den Risikokategorien in der Modellierung von Bagust et al. überein, sondern basiert auf einer Reanalyse der Registerdaten auf Wunsch des „Appraisal Committee“, das die Leitlinienempfehlungen abgibt (NICE 2008). Die in der Empfehlung genannte Subgruppe stimmt mit der Subgruppenempfehlung der Vorgänger-Leitlinie von 2003 überein. Neben der Beschränkung auf eine bestimmte Patientengruppe wurde in der neuen Leitlinie auch ein Maximum des Mehrpreises für DES festgesetzt und als Mittel zur Gewährleistung eines kosteneffektiven Einsatzes verwendet.

Tab. 27 IKER für Subgruppen in der Leitlinie von NICE (2008)

\begin{tabular}{|lcc} 
& Differenz DES - BMS & Differenz DES - BMS \\
& $400 €$ & $800 €$ \\
\hline alle Patienten & $98.000 € /$ QALY & $227.000 € /$ QALY \\
\hline Patienten Läsionen $>15 \mathrm{~mm}$ & $62.000 € /$ QALY & $167.000 € /$ QALY \\
\hline Patienten Gefäße $<3 \mathrm{~mm}$ & $33.000 € /$ QALY & $126.000 € /$ QALY \\
\hline
\end{tabular}




\subsection{Diskussion}

Die Erstellung von KSLL ist möglich und findet in der Praxis in anderen Ländern bereits statt. Am Beispiel von DES lässt sich gut zeigen, dass es allerdings einige methodische Herausforderungen gibt, die zum einen mit dem Vorhandensein und der Generierung empirischer Evidenz zu tun haben, wie dies für alle Bereiche der evidenzbasierten Medizin gilt, und zum anderen werden im Prozess der Erstellung von KSLL Werturteile gefällt, beispielsweise bei der Abwägung zwischen Nutzen und Risiken, die nicht allein Sache der Wissenschaft sind.

\subsubsection{Besondere methodische Aspekte bei der Erstellung von KSLL}

Der Nachweis der klinischen Effektivität ist Voraussetzung für alle Kosteneffektivitätsbetrachtungen und muss nach den Methoden der evidenzbasierten Medizin durchgeführt werden.

\section{Subgruppenanalysen}

Nicht nur für eine nachfolgende ökonomische Analyse kann die Stratifizierung in Subgruppen mit größerem und geringerem Zusatznutzen durch eine neue Technologie wichtig sein. Wie am Beispiel von DES gut zu sehen ist, ist dies auch bei Nutzen-Schaden-Abwägungen der Fall. Für den Wirksamkeitsaspekt können Subgruppenanalysen bereits bei der Planung von RCT berücksichtigt werden. Dies erfordert größere Fallzahlen und die Beachtung entsprechender Analysestandards für die Auswertung (MDS 2004). Die Subgruppenanalyse von unerwünschten Nebenwirkungen ist wegen der geringen Häufigkeit in der Regel schwieriger, weil hier größere Stichproben erforderlich sind und vor der Einführung einer Technologie nicht unbedingt klar sein muss, welche Schäden auftreten werden. Metaanalysen von RCT und Analysen von Registerdaten sind hier in der Regel geeignete Hilfsmittel. Durch Metaanalysen ließ sich im Fall von DES die Häufigkeit von späten Stentthrombosen relativ früh abschätzen und quantifizieren. Die Ergebnisse zur Wirksamkeit und Sicherheit in systematischen Übersichtsarbeiten und evidenzbasierten Leitlinien sollten zu gemeinsamen Effektschätzern mit Angaben zur statistischen Unsicherheit für die gesamte Studienpopulation und die Subgruppen zusammengefasst werden, damit Effektivität und Sicherheit zwischen den Subgruppen verglichen werden kann. Voraussetzung für solche Metaanalysen ist eine ausreichende Homogenität der Studien. Diese Effektschätzer können dann auch in gesundheitsökonomischen Modellierungen verwendet werden. 


\section{Übertragbarkeit}

Um zu Praxisempfehlungen zu kommen, müssen klinische Leitlinien abschätzen, inwiefern die Effekt- und Sicherheitsdaten in den RCT auf die Population im klinischen Alltag übertragbar sind. Entsprechende ergänzende Daten aus der Versorgungsforschung sind deshalb wichtig. Am Beispiel von DES ist gut zu sehen, dass sich die Kosteneffektivität stark verändern kann, wenn die Zusammensetzung der Studienpopulation hinsichtlich der prognostischen Faktoren stärker von der Zielpopulation abweicht. Epidemiologische Unterschiede bei der Zielerkrankung oder eine unterschiedliche Versorgungspraxis können hier ebenfalls eine Rolle spielen wie deutliche Unterschiede in der Häufigkeit von PCI pro Einwohner in Europa zeigen. Um Kostenaspekte und die Kosteneffektivität analysieren zu können, müssen landesspezifische Kosteneffektivitätsstudien zur Verfügung stehen. Register sollten den Ressourcenverbrauch und Preise mit erheben, wie dies z.B. bei einigen kardiovaskulären Registern bereits getan wird (z.B. STAR-Register zur Therapie von Patienten mit stabiler Angina pectoris oder das Deutsche Aortenklappenregister). Häufig werden auch Modellierungsstudien erforderlich sein, um sich ändernde Parameter wie neue Effektivitätsdaten oder sich ändernde Kosten einfach berücksichtigen zu können und durch Sensitivitätsanalysen die Faktoren mit dem stärksten Einfluss auf die Kosteneffektivität zu identifizieren oder internationale Daten auf den deutschen Kontext zu übertragen (Siebert 2003, 2005).

\section{Werturteile in KSLL}

Am Beispiel von DES zeigt sich, dass bereits die Nutzen-Schaden-Abwägung stark von Präferenzen abhängig ist. Die wissenschaftliche Analyse kann zwar ihren Beitrag zur Entscheidungsfindung leisten, indem sie möglichst genaue Abschätzungen der Risiken liefert und diese verständlich kommuniziert. Ob aber beispielsweise ein leicht erhöhtes Risiko für eine Stentthrombose bei Patienten mit kleinen Gefäßen und langen Läsionen oder die erhöhte Blutungsgefahr durch die verlängerte Clopidogrelgabe für den potenziell größerer Nutzen in Kauf genommen wird oder nicht, hängt von der Risikobereitschaft von Arzt und Patient ab, vorausgesetzt, dass der Patient ausreichend aufgeklärt wurde. Leitlinien setzen hier mit ihren Empfehlungen einen gewissen Rahmen.

Bei KSLL kommt die Wertentscheidung über die gesellschaftliche Zahlungsbereitschaft für die jeweiligen Interventionen hinzu. Diese Entscheidung kann jedoch nicht auf der individuellen Ebene getroffen werden, sondern ist von der gesellschaftspolitischen Konsensfindung und rechtlichen Rahmenbedingungen abhängig. Konsistente Empfehlungen im Rahmen von KSLL sind erst möglich, wenn diese Punkte geklärt sind (s. Kap. 2). 


\section{Limitationen}

Im Rahmen des Projekts zur Ressourcenallokation war es nicht möglich, die gesamte Evidenz zu DES aufzuarbeiten, da die Menge an Material im zeitlichen und finanziellen Rahmen des Projekts nicht zu bewältigen war. Die angeführte Evidenz ist nicht vollständig und mittlerweile überholt. Reale Empfehlungen zu DES können anhand der Auswahl der hier dargestellten Studien nicht abgeleitet werden. Prinzipielle methodische Fragen konnten jedoch aufgezeigt werden.

Nicht näher betrachtet wurde hier die Frage, welche Technologien und welche Indikationen bei einem bestimmten Krankheitsbild vergleichend zu betrachten sind. So können sich durch die Einführung von Technologien Indikationsverschiebungen ergeben, entweder aus Sicherheitsgründen oder weil die erhöhte Effektivität neue Möglichkeiten eröffnet: Zum Beispiel könnten DES für Patienten, die bisher nur mit einer Bypass-Operation versorgt werden konnten, infrage kommen. Umgekehrt zeigen andere Studien, die PCI und medikamentöse Behandlung vergleichen, dass die Indikation zur PCI ggf. einzuschränken ist (Wijeysundera et al. 2010). Grundsätzlich müssten alle relevanten medizinischen Technologien für ein Krankheitsbild in KSLL verglichen werden, um bestimmen zu können, was die besten und kosteneffektivsten Therapien für das jeweilige Krankheitsbild sind.

\section{Literatur}

Bagust A, Grayson AD, Palmer ND, Perry RA, Walley T. Cost effectiveness of drug eluting coronary artery stenting in a UK setting: cost-utility study. Heart 2006;92(1):68-74.

Bakhai A, Stone GW, Mahoney E, Lavelle TA, Shi C, Berezin RH, et al. Cost Effectiveness of Paclitaxel-Eluting Stents for Patients Undergoing Percutaneous Coronary Revascularization: Results From the TAXUS-IV Trial. 2006;48(2):253-61

Bowen I, Hopkins R, He Y, Blackhouse G, Lazzam C, Tu I, et al. Systematic review and cost-effectiveness analysis of drug eluting stents compared to bare metal stents for percutaneous coronary interventions in Ontario. Interim report for the Ontario Ministery of Health and Long-term Care. Program for Assessment of technology in Health 2005:170.

Bruckenberger E. 19. Herzbericht 2006 mit Transplantationschirurgie: 19. Bericht Sektorenübergreifende Versorgungsanalyse zur Kardiologie und Herzchirurgie in Deutschland sowie vergleichende Daten aus Österreich und der Schweiz. Hannover 2007.

Cohen DI, Bakhai A, Shi C, Githiora L, Lavelle T, Berezin RH, et al. Cost-effectiveness of Sirolimus-eluting stents for treatment of complex coronary stenoses: Results from the sirolimus-eluting balloon expandable stent in the treatment of patients with de novo native coronary artery lesions (SIRIUS) trial. Circulation 2004;110(5):508-14.

Ekman M, Sjogren I, James S. Cost-effectiveness of the Taxus paclitaxel-eluting stent in the Swedish healthcare system. Scand-Cardiovasc-J 2006;40(1):17-24.

Elezi S, Dibra A, Folkerts U, Mehilli J, Heigl S, Schomig A, et al. Cost Analysis From Two Randomized Trials of Sirolimus-Eluting Stents Versus Paclitaxel-Eluting Stents in High-Risk Patients With Coronary Artery Disease. 2006;48(2):262-7.

Farb A, Boam AB Stent thrombosis redux - the FDA perspective. N Engl I Med 2007;356:984-7 
FDA. Summary from the Circulatory System Devices Panel Meeting - December $7 \&$ 8, 2006. URL: http://www. fda.gov/AdvisoryCommittees/CommitteesMeetingMaterials/MedicalDevices/MedicalDevicesAdvisoryCommittee/CirculatorySystemDevicesPanel/ucm124639.htm (Zugriff am 21.04.2015)

Gorenoi V, Dintsios CM, Hagen A. Coated stents to prevent restenosis in coronary heart disease. GMS Health Technol Assess. 2005;1:Doc06.

Greenberg D, Bakhai A, Cohen DI. Can we afford to eliminate restenosis?: Can we afford not to? I-Am-Coll-Cardiol 2004;43(4):513-8.

Greenberg D, Cohen D). Examining the economic impact of restenosis: implications for the cost-effectiveness of an antiproliferative stent. Z-Kardiol 2002;91 Suppl 3:137-43.

Hill R, Bagust A, Bakhai A, Dickson R, Dündar Y, Haycox A, et al. Coronary artery stents: a rapid systematic review and economic evaluation. Health Technol Assess 2004;8(35).

Hodgson IM, King SB 3rd, Feldman T, Cowley MJ, Klein LW, Babb ID; Society for Cardiac Angiography and Interventions. SCAI Statement on Drug-Eluting Stents: Practice and Health Care Delivery Implications. Catheterization Cardiovascular Interventions 2003;58: 397-9.

Janatzek S, Arndt C, Windeler J. MDS-Medizinischer Dienst der Spitzenverbände der Krankenkassen e.V. G-2 Gutachten: Aussagekraft von Subgruppenanalysen. 2004

Kaiser C, Brunner La Rocca HP, Buser PT, Bonetti PO, Osswald S, Linka A, et al. Incremental cost-effectiveness of drug-eluting stents compared with a third-generation bare-metal stent in a real-world setting: Randomised Basel Stent Kosten Effektivitats Trial (BASKET). Lancet 2005;366(9489):921-9.

Kaiser C, Brunner La Rocca HP, Buser PT. Erratum: Incremental cost-effectiveness of drug-eluting stents compared with a third-generation bare-metal stent in a real-world setting: Randomised Basel Stent Kosten Effektivitats Trial (BASKET) (Lancet [2005] 366 [921-929]). Lancet 2005;366(9503):2086.

King SB 3rd, Smith SC, Morrison DA, Williams D0 et al. Focused Update of the ACC/AHA/SCAI 2005 Guideline Update for Percutaneous Coronary Intervention. A Report of the American College of Cardiology/American Heart Association Task Force on Practice Guidelines. Journal of the American College of Cardiology 2008;51(2):172-209.

Medizinischer Dienst der Spitzenverbände der Krankenkassen e.V., Fachbereich Evidenz-basierte Medizin: G-2 Gutachten: Aussagekraft von Subgruppenanalysen, 2004. URL: http://www.mds-ev.de/media/pdf/Gutachten_Subgruppenanalysen.pdf (Zugriff am 21.04.2015)

Moses JW, Leon MB, Popma II, Fitzgerald PI, Holmes DR, O'Shaughnessy C, Caputo RP, Kereiakes DI, Williams DO, Teirstein PS, Jaeger IL, Kuntz RE; SIRIUS Investigators. Sirolimus-eluting stents versus standard stents in patients with stenosis in a native coronary artery. NEJM 2003;349:1315-23

NICE, NHS. Guidance on the use of coronary artery stents. NICE technology appraisal guidance 2003;71.

NICE, NHS. Drug-eluting stents for the treatment of coronary artery disease. Part review of NICE technology appraisal guidance 71. NICE technology appraisal guidance 2008;152.

Organisation for Economic Co-operation and Development (OECD). Prices and purchasing power parities. URL: http://stats.oecd.org/index.aspx?DataSetCode=PPPGDP\# (Zugriff am 21.04.2015)

Ruygrok PN, Melkert R, Morel M-AM, Ormiston JA, Bär FW, Fernandez-Avilès F, et al. Does angiography six months after coronary intervention influence management and outcome? I Am Coll Cardiol 1999;34:150711.

Ryan I, Cohen DJ. Are Drug-Eluting Stents Cost-Effective?: It Depends on Whom You Ask. Circulation. 2006;114(16):1736-44.

Schnell-Inderst P, Hessel F, Siebert U, Klauss V, Wasem I. Medizinische und ökonomische Evaluation Medikamente-freisetzender Stents bei perkutanen Koronarinterventionen im Vergleich zu konventionellen Stents und Bypassoperationen - ein Health Technology Assessment, 2005. URL: http://www.mm.wiwi.uni-due. de/fileadmin/fileupload/BWL-MEDMAN/Forschung/DESHTA.pdf (Zugriff am 21.04.2015)

Schöffski 0, Schumann A. Das Schwellenwertkonzept. In: Schoffski 0, Graf v.d. Schulenburg J-M (Hrsg.). Gesundheitsökonomische Evaluation. Vol. 3rd. Berlin, Heidelberg, New York: Springer. 2008;139-66.

Shrive FM, Ghali WA, Lewis S, Donaldson C, Knudtson ML, Manns BJ. Moving beyond the cost per quality-adjusted life year: Modelling the budgetary impact and clinical outcomes associated with the use of sirolimuseluting stents. Can---Cardiol 2005a;21(9):783-87. 
Shrive FM, Manns B], Galbraith PD, Knudtson ML, Ghali WA. Economic evaluation of sirolimus-eluting stents. Can-Med-Assoc-1 2005b;172(3):345-51.

Siebert U. [Using decision-analytic modelling to transfer international evidence from health technology as sessment to the context of the German health care system. A HTA methods report. Series of the German Institute for Medical Documentation and Information commissioned by the Federal Ministry of Health and Social Security]. Cologne: DIMDI; 2005.

Siebert U. When should decision-analytic modeling be used in the economic evaluation of health care? [Editorial]. European Journal of Health Economics. 2003;4(3):143-50.

Silber S, Albertsson P, Avilés FF, Camici PG, Colombo A, Hamm C, |ørgensen E, Marco |, Nordrehaug |E, Ruzyllo W, Urban P, Stone GW, Wijns W; Task Force for Percutaneous Coronary Interventions of the European Society of Cardiology. Guidelines for Percutaneous Coronary Interventions. The Task Force for Percutaneous Coronary Interventions of the European Society of Cardiology. Eur Heart I. 2005;26(8):804-47.

Silber S, Borggrefe M, Böhm M, Hoffmeister HM, Dietz R, Ertl G, Heusch G. Positionspapier der DGK zur Wirksamkeit und Sicherheit von Medikamente freisetzenden Koronarstents (DES). Eine evidenzbasierte Analyse von 71 randomisierten Studien mit 28.984 Patienten. Der Kardiologe 2007;1:84-111

Smith SC Ir, Feldman TE, Hirshfeld JW Jr, Jacobs AK, Kern MJ, King SB 3rd, Morrison DA et al. ACC/AHA/SCAI 2005 Guideline Update for Percutaneous Coronary Intervention. A Report of the American College of Cardiology/ American Heart Association Task Forde on Practice Guidelines (ACC/AHA/SCAI Writing committee to Update the 2001 Guidelines for Percutaneous Coronary Intervention). I Am Coll Cardiol. 2006;47(1):e1-121.

Stone GW, Ellis SG, Cox DA, Hermiller I, O'Shaughnessy C, Mann JT, Turco M, Caputo R, Bergin P, Greenberg I, Popma II, Russell ME; TAXUS-IV Investigators. One-year clinical results with the slow-release, polymer-based, paclitaxel-eluting TAXUS stent. The TAXUS IV trial. Circulation 2004;109(16):1942-7.

Szucs TD, Schwenkglenks M, Lüscher TF, Eberli F. Die Wirtschaftlichkeit beschichteter Stents in der interventionellen Kardiologie - eine gesamtschweizerische Analyse. Kardiovaskuläre Medizin 2005;8:41-52.

Tarricone R, Marchetti M, Lamotte M, Annemans L, De Jong P. What reimbursement for coronary revascularization with drug-eluting stents? Eur--Health-Econ 2004;5(4):309-16.

Wijeysundera HC, Nallamothu BK, Krumholz HM, Tu JV, Ko DT. Meta-analysis: Effects of percutaneous coronary intervention versus medical therapy on angina relief. Ann Intern Med 2010;152(6):370-9. 